\title{
Effect of a Local Axisymmetric Imperfection on the Buckling Behavior of a Circular Cylindrical Shell under Axial Compression
}

\author{
J. W. Hutchinson* \\ Harvard University, Cambridge, Mass. \\ AND \\ R. C. Tennyson $\dagger$ AND D. B. MUgGeridge $\ddagger$ \\ University of Toronto, Toronto, Ontario, Canada
}

\begin{abstract}
A combined theoretical and experimental investigation has been carried out on the effects of certain types of local axisymmetric imperfections on the buckling of cylindrical shells under axial compression. Buckling loads have been calculated for a variety of "dimple" imperfections. Results have been obtained for constant thickness shells with middle surface variations from the geometry of a perfect eylinder as well as for shells with local axisymmetrie thickness variations. Nonlinear prebuckling effects and edge conditions are taken into account. In the experimental program a series of seven photoelastic plastic circular cylindrical shells each containiug a local axisymmetric dimple centered at mid-length were tested under pure axial compression. All cylinders were constructed by the spin-casting technique and the local imperfection was cut on both the inner and outer walls using a hydraulic tracer-tool apparatus in conjunction with a metal template. A broad range of imperfection amplitudes and wavelengths was investigated. The experimental results were in good agreement with the theoretical predictions.
\end{abstract}

\section{Nomenclature}

$C=\left[3\left(1-z^{2}\right)\right]^{1 / 2}$

$E=$ modulus of elasticity

$L=$ shell length

$l_{x}=$ axial half wavelength of dimple

$l_{c}=\pi\left[12\left(1-x^{2}\right)\right]^{-1 / 4}(R i)^{1 / 2} ;$ half wavelength of classical axisymmetric buckling mode

$R$ = shell radius measured to the middle surface

$i=$ shell wall thickness

$\bar{i}=$ average wall thickness

$l^{*}=$ average wall thickness mensured over only dimple area

$w=$ radial displacement-positive outward

$x=$ axial coordinate with origin at the shell mid-length

$\tilde{x}=\pi x / l_{c}$

$Z=\left(1-y^{2}\right)^{1 / 2} L^{2} / R t$

$\beta=l_{x} / l_{c}$

$\delta=$ peak amplitude of axisymmetric middle surface imperfection

$\Delta=\operatorname{sec~Lq} \cdot(1)$

$\lambda_{\theta}=2 \pi E t^{2} / C$; classical buckling load of an infinitely long cylindrical shell under axial compression

$\lambda^{*}=$ buckling load (i.e., maximum support load of imperfect shell)

$\mu=$ maximum deviation of median surface/average wall thickness

$\nu=$ Poisson's ratio

Presented as Paper 70-103 at the AIAA 8th Aerospace Sciences Meeting, New York, January 19-21, 1970; revision received May 4,1970 . This work was supported in part by the National Research Council of Canada (NRC Grant No. A-2783), the National Aeronantics and Space Administration [NASA Grant No. 52-026-(011); also NASA Grant NGL 22-007-012] and the Division of Lingineering and Applied Physics, Harvard University.

* Professor of Applied Mechanics, Division of Engineering and Applied Physics. Member ATAA.

f Associate Professor, Institute for Aerospace Studies. Member AIAA.

$\ddagger$ Research Assistant, Institute for Aerospace Studies.

\section{Introduction}

$\mathbf{T}$ THE presence of shape imperfections in circular cylindrical shells subjected to axial compressive loading has now been recognized as the dominant factor in reducing the buckling load significantly below the classical value. Although the effects of end constraint do lower buckling loads, it has been shown both theoretically ${ }^{1}$ and experimentally ${ }^{2}$ that the clamped case, which is perhaps most often encountered in practice particularly in the laboratory, accounts for nominally a $10 \%$ reduction. As demonstrated recently, a uniform distribution of axisymmetric imperfections having the form of a simple trigonometric function drastically reduces the shell buckling load. In particular it was found that the load reduction was dependent both on the imperfection amplitude and wavelength, and could be accurately predicted using Koiter's extended theory, Other investigators, ${ }^{4,5}$ have also demonstrated experimentally that imperfections significantly affect the buckling behavior of circular cylindrical shells under axial compression. Although imperfection distributions are likely to be random in nature, it is often observed that local dimples or shape imperfections are present in the shell structure. Recently, the effects of local cutouts ${ }^{6,7}$ and local circular dimples on the buckling of circular cylinders have been studied and have been found to give rise to low buckling loads.

Here, a number of theoretical and experimental results are presented on the effects of local axisymmetric imperfections on the buckling behavior of cylindrical shells under axial compression. Shells with various dimple forms are studied including constant thickness shells with initial middle surface deviations as well as shells with variable thickness and associated middle surface variations.

\section{Asymptotic and Numerical Predictions}

Amazigo and Budiansky ${ }^{9}$ have obtained a general formula for the buckling load of an infinitely long cylindrical shell under axial compression containing an arbitrary, localized axi- 

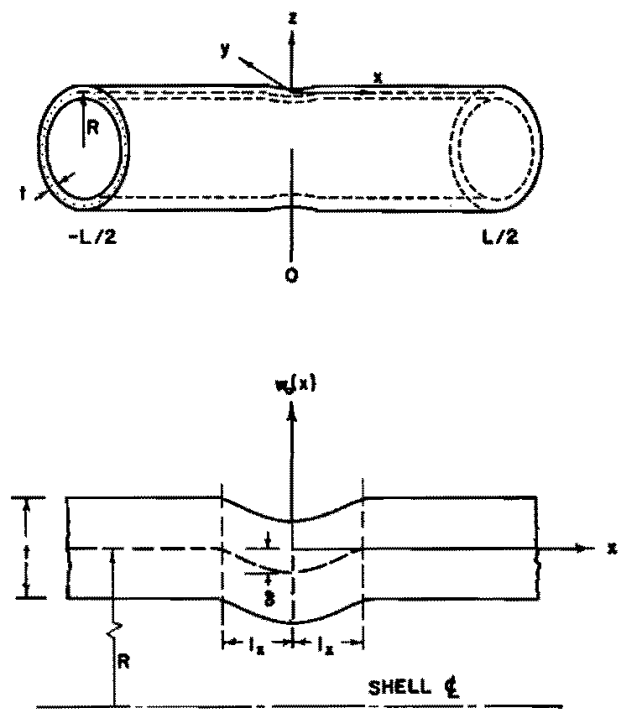

$y(x)=-\frac{g}{2}\left(1+\cos \frac{r x}{1 x}\right) ;-1, \leq x \leq 1 x$

Mal 00 , FOR ALL OTHER VALUES OF *

Fig. I Circular cylindrical shell of constant thickness with inward axisymmetric dimple imperfection in shape.

symmetric imperfection. Their formula is an asymptotic one which is valid for sufficiently small imperfections in much the same way as is Koiter's formula for a sinusoidal axisymmetric imperfection. ${ }^{10,11}$

The initial axisymmetric imperfection $w_{0}$ in the middle surface (assumed to have a continuous slope) of a cylinder of constant thickness $t$ enters into the buckling formula through

$$
\Delta=\int_{-\infty}^{\infty} \frac{w_{0}}{t} e^{i x} d \tilde{x}
$$

where $x=\pi x / l_{c}$ and $l_{\varepsilon}=\pi\left[12\left(1-\nu^{2}\right)\right]^{-1 / 4}(R t)^{1 / 2}$ is the half wavelength of the classical axisymmetric buckling mode. The asymptotic formula of Ref. 9 for the ratio of the buckling load $\lambda^{*}$ to the classical buckling load $\lambda_{c}$ is

$$
\left(1-\lambda^{*} / \lambda_{c}\right)^{8 / 2}=\left[3 C /\left(2^{3 / 2}\right)\right]|\Delta|\left(\lambda^{*} / \lambda_{c}\right)
$$

where $C=\left[3\left(1-v^{2}\right)\right]^{1 / 2}$.

Of primary interest here is a "cosine dimple" defined by

$$
\begin{aligned}
w_{0} & =-\delta / 2\left(1+\cos \pi x / l_{x}\right) & & |x| \leq l_{x} \\
& =0 & & |x|>l_{x}
\end{aligned}
$$

so that $l_{x}$ is the half length of the dimple. In this case,

$$
\Delta=\left[\sin (\pi \beta) / \beta^{2}-1\right](\delta / l)
$$

where $\beta \equiv l_{x} / l_{c} . \quad$ When $\beta=1$, Eq. (4) reduces to

$$
|\Delta|=2 \pi(\delta / t)
$$

The maximum reduction in the buckling load, for a given imperfection amplitude-thickness ratio $\delta / t$, occurs when $\beta \cong 0.8$ with $\Delta$ about $5 \%$ larger than $\mathrm{Eq} .(5)$.

Numerical calculations have also been carried out for finite length shells with the cosine dimple, Eq. (3), symmetrically located with respect to the ends of the shell as shown in Fig. 1. The calculation procedure, which has been described in Ref. 3 , takes into account end effects and nonlinear prebuckling deformations. The prebuckling problem and the reduced eigenvalue problem are both obtained without approximation from a Karman-Donnell-type shell theory. Resulting ordinary differential equations are cast in finite difference form and solved by a Gaussian elimination method usually referred to as Potters' method. Clamped end conditions are chosen for all the results presented here since they pertain most closely to those in the testing program.

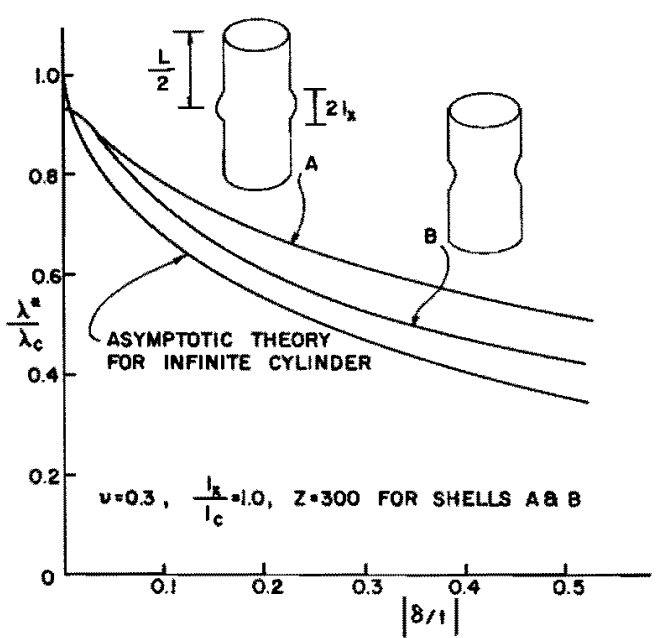

Fig. 2 The effect of an axisymmetrical dimple imperfeetion on the buckling of clamped eylindrical shells (constant thickness) under axial compression.

Predictions based on the asymptotic formula [Eq. (2)] and the numerical calculations are compared in Fig. 2 for $l_{x} / l_{t}=1$. Only the absolute value of the imperfection amplitude enters into the asymptotic formula and thus the curve shown holds for inward and outward dimples. On the other hand, the numerical results bring out some difference between the buckling load for a bulge (curve $A$ ) and a pinch (curve $B$ ) with the latter having the more degrading effect. Agreement between the asymptotic formula and the numerical results is quite good except, of course, for extremely small imperfection amplitudes in which case end effects dominate the buckling behavior of finite length shells. The length parameter $Z$ for these shells was taken to be 300 , so that for all practical purposes the buckling load is independent for values of $Z$ in this range and greater. (All calculations were made with 120 finite difference stations over the half length of the shell and isolated results were checked using 250 stations.)

Effects of local thickness variations are illustrated by the results presented in Fig. 3. These results were also obtained numerically in the manner described previously and detailed further in Ref. 3. For both the lowest and uppermost curves the thickness variation is given by

$$
\begin{aligned}
\Delta t & =\delta\left(1+\cos \pi x / l_{x}\right) & & |x| \leq l_{x} \\
& =0 & & |x|>l_{x}
\end{aligned}
$$

with either the inner or outer wall unperturbed as shown. Thus, this variation gives rise to exactly the same initial mid-

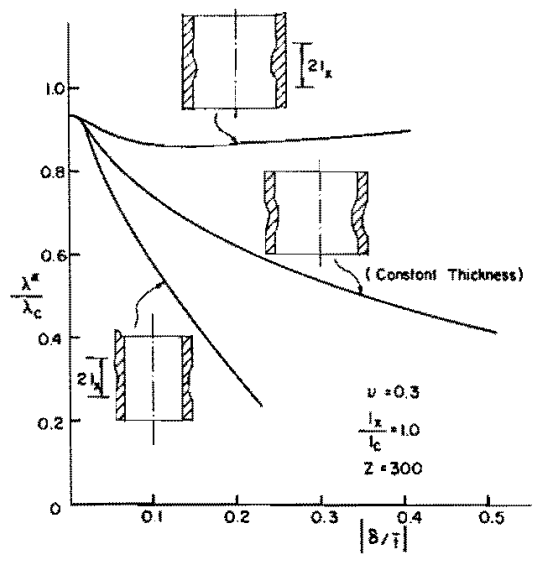

Fig. 3 A comparison of the effects of different thickness variations on the buckling of clamped cylindrical shells under axial compression. 


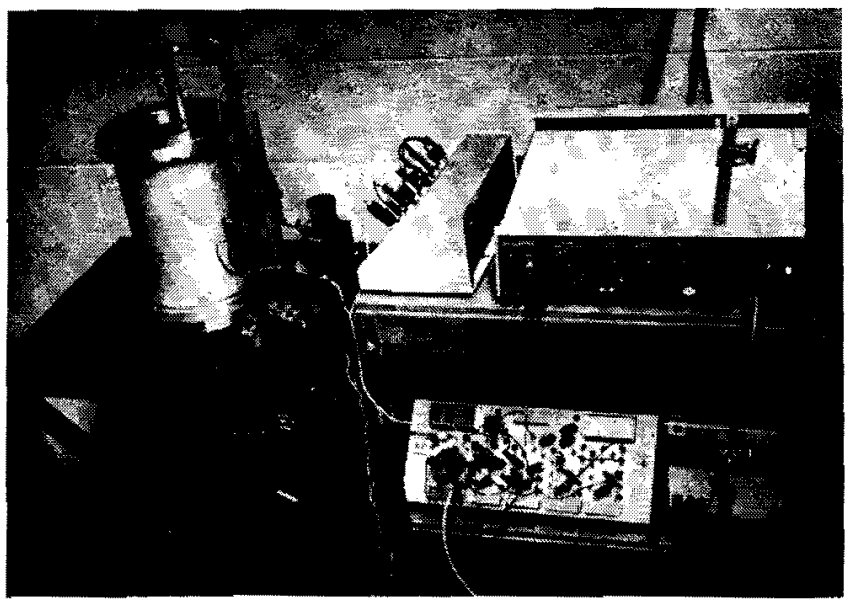

Fig. 4 Cylinder profile measuring apparatus.

dle surface variation as described by Eq. (3). It is not surprising that a shell which has both an inward middle surface variation and a reduction in its thickness buckles at a load considerably below a constant thickness shell with an identical middle surface variation. Even a thickening of the shell, as indicated by the uppermost curve, results in a small reduction in the buckling load if $\delta / t$ is sufficiently small.

Localized dimples in constant thickness cylindrical shells have an effect which is somewhat less severe than a sinusoidal axisymmetric imperfection. Almroth ${ }^{12}$ has made the same observation on the basis of his studies of the effects of axisymmetric imperfections on cylindrical shells. Axisymmetric dimple studies of conical shells lead to similar conclusions, ${ }^{13}, 14$

\section{Experiment-Fabrication and Test Procedure}

All test cylinders were made by the spin-casting technique? using a photoelastic liquid epoxy plastic. Initially, a geo-

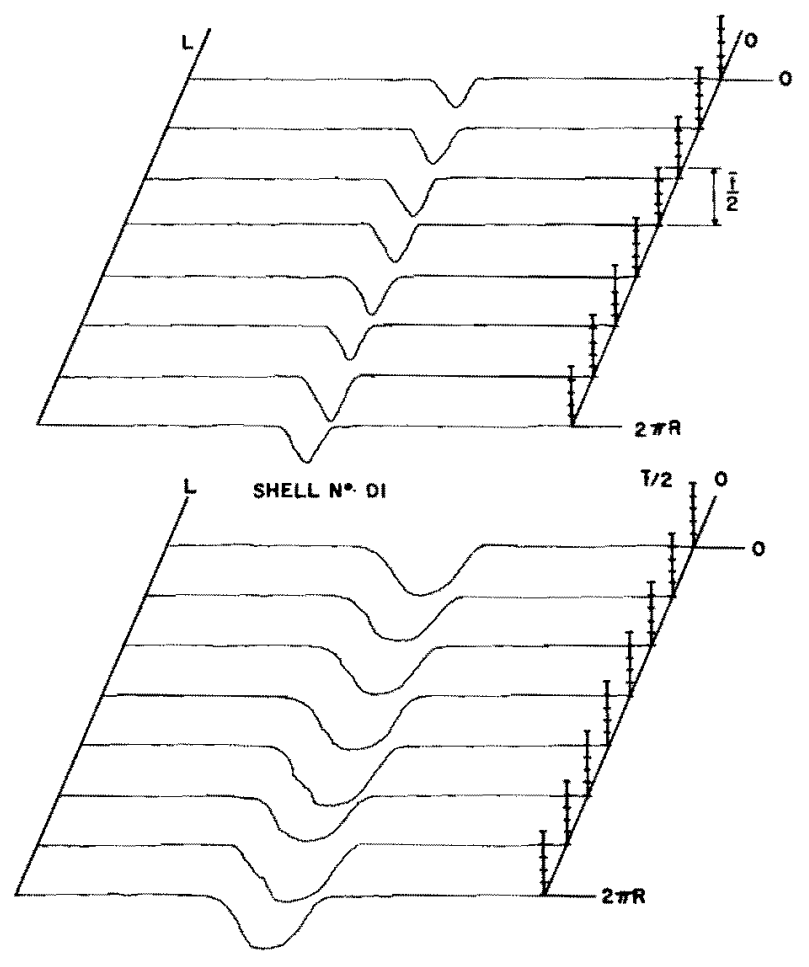

SHEL N* D2

Fig. 5 Profiles of the median surface of circular cylindrical shells of constant thickness containing axisymmetric dimple imperfections in shape. metrically near-perfect circular cylindrical shell was cast and the inner wall machined to the prescribed profile with an axisymmetric dimple centered at mid-length. Subsequently, the cylinder was removed from the form and thickness measurements were made at discrete points around the circumference at both ends of the shell. Machined aluminum end plates were then attached to the test eylinder to provide the clamped edge constraint. To obtain a constant thickness shell, the outer surface was machined to the same profile as the inner wall by inserting into the cylinder a ring stiffener located at the dimple to reinforce the shell wall during the machining stage. Each imperfection shape was constructed using a metal template containing the desired amplitude and wavelength in conjunction with a hydraulic tracer-tool apparatus. In order to determine the actual inner and outer shell wall profile, each model was placed in a rotation apparatus (Fig. 4) and axial contours were obtained using two low pressure, linear contacting displacement transducers, with their outputs recorded on an $x-y$ plotter. Figure 5 illustrates median surface profiles determined from two of the test cylinders. It is quite evident that the dimples are axisymmetric i.e., little circumferential variation, and centered at mid-length. A comparison of the dimple shape with the assumed wave form given by $\mathrm{Eq}$. (3) is shown in Fig. 6 using a randomly selected generator from each test cylinder. It can be readily seen that the assumed and actual profiles are in excellent agreement. A summary of the shell properties is contained in Table 1. Of particular significance is the difference in values between the average shell wall thickness $\bar{t}$ and the average shell wall thickness measured only over the dimple area, denoted by $\vec{t}^{*}$. This variation appreciably affects the values of $\lambda^{*} / \lambda_{c}, \mu$ and $\beta$ which are used in the comparison with the exact model results, as will be discussed later.

The final stage of the test procedure involved the proper alignment of the cylinder in an electrically driven, four-screw compression machine. Uniformity of the applied stress was easily checked by examining photoelastically the membrane stress distribution around the circumference of the cylinder and finally, by noting the postbuckled configuration of the cylinder. Complete circumferential buckling centrally located either in the dimple (one tier) or bounding the dimple (two tiers) attested to the uniformity of the load distribution. To serve as a reference shell, a geometrically near-perfect

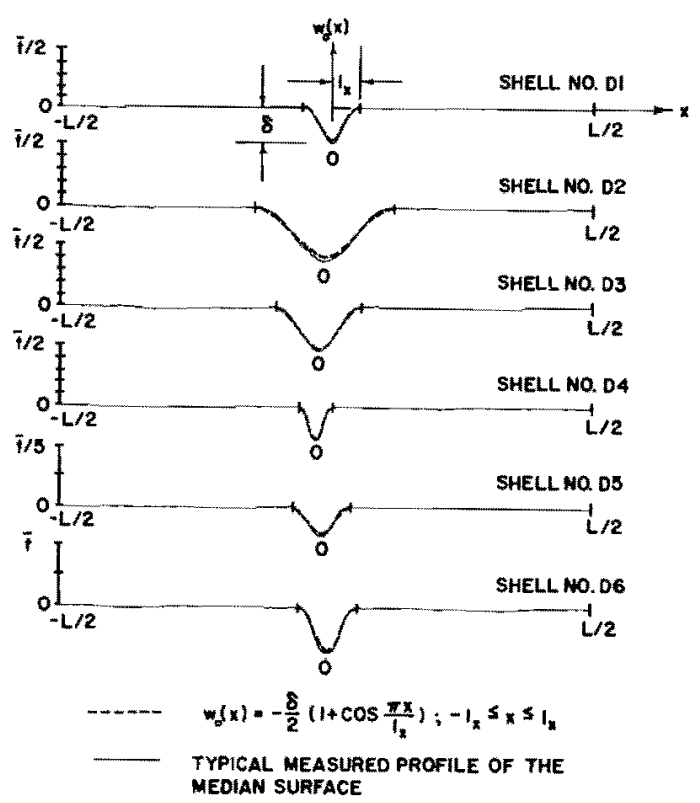

Fig. 6 Typical profiles of the median surface of circular cylindrical shells of constant thickness containing axisymmetric dimple imperfections in shape. 
Table 1 Properties of shells containing an axisymmetrie dimple

\begin{tabular}{|c|c|c|c|c|c|c|c|c|c|}
\hline \multirow{2}{*}{$\begin{array}{l}\text { Shell } \\
\text { No. }\end{array}$} & \multicolumn{6}{|c|}{ IN } & \multirow[b]{2}{*}{$\mu$} & \multirow[b]{2}{*}{$l_{x} / l_{c}$} & \multirow[b]{2}{*}{$\left(\lambda^{*} / \lambda_{c}\right)_{\theta \times p}$} \\
\hline & $R$ & $l$ & $l^{*}$ & $L$ & $\delta$ & $l_{x}$ & & & \\
\hline D1 & 3.92 & 0.0211 & 0.0242 & 11.0 & 0.0070 & 0.550 & 0.289 & 1.02 & 0.473 \\
\hline D2 & 3.92 & 0.0189 & 0.0188 & 11.0 & 0.0069 & 1.375 & 0.367 & 2.87 & 0.857 \\
\hline D3 & 3.92 & 0.0194 & 0.0192 & 11.0 & 0.0075 & 0.834 & 0.391 & 1.72 & 0.629 \\
\hline D4 & 3.92 & 0.0183 & 0.0188 & 11.0 & 0.0062 & 0.306 & 0.330 & 0.64 & 0.449 \\
\hline D5 & 3.92 & 0.0207 & 0.0215 & 11.0 & 0.0022 & 0.550 & 0.102 & 1.14 & 0.714 \\
\hline D6 & 3.92 & 0.0224 & 0.0236 & 11.0 & 0.0173 & 0.550 & 0.734 & 1.03 & 0.345 \\
\hline $\begin{array}{l}\mathrm{D} 7 \\
\mathrm{E}=3.94 \\
y=0.40 \\
\text { Note: } \mu, l\end{array}$ & $\begin{array}{l}3.92 \\
\times 10^{*} \mathrm{PSI} \\
\\
{ }_{*} / l_{c}, \lambda^{*} / \lambda_{c}\end{array}$ & 0.0179 & $\ldots$ & 11.0 & $\ldots$ & . & $\ldots$ & $\cdots$ & 0.919 \\
\hline
\end{tabular}

cylinder was tested in axial compression to determine the modulus of the elasticity of the epoxy plastic used in the test series, and at the same time, provide a measure of the load reduction due to the clamped edge constraint common to each shell. In total, seven cylinders of varying imperfection wavelength and amplitude were studied (refer to Table 1), the results of which are discussed in the next section.

\section{Discussion of Experimental Results}

A comparison of shell buckling data for shells with inward dimples and theoretical predictions from both the asymptotic formula [Eq. (2)] and the numerical calculations is made in Fig. 7. The theoretical curves have been obtained using $l_{x} / l_{c}=1.06$ which is the average of the corresponding values for the three test specimens. As alluded to previously, the average wall thickness $t^{*}$, which was measured only over the dimple area defined by $|x| \leq l_{x}$, was used as the reference thickness in calculating the buckling loads. Justification of this step rests on the argument that buckling is a local phenomenon governed by the dimpled region. In any case, the discrepancy between the average shell thickness $\bar{t}$ and the local average $t^{*}$ was less than five percent in every shell but one as listed in Table 1. All the test specimens fatl in the range in which the numerical predictions are essentially independent of the shell length.

The agreement between test and theory, particularly with the numerical results, is very good, especially so considering the long history of discrepancy between test and theory for such structures. Equally good comparisons have recently been obtained for the effects of sinusoidal imperfections. ${ }^{3}$
Figure 8 displays plots of buckling loads for constant thickness shells with inward dimples over a range of values of the imperfection wavelength ratio $l_{r} / l_{\varepsilon}$ for a constant value of the imperfection-thickness ratio $\delta / t$. The value of $\delta / t=0.363$ was chosen as the average of the values for the three experimental points plotted. It is clear that the critical half wavelength occurs for $l_{x} / l_{c} \cong 0.8$ and the associated buckling load is only a few percent below the predicted value for $l_{x} / l_{t}=1$. The asymptotic formula shows the same general trend as the numerical results. Namely, when the ratio $l_{x} / l_{c}$ is a factor 3 , say, greater or less than unity, the effect of the imperfection is much less than at the critical value of $l_{z} / l_{c}$. The experiments show this very clearly.

\section{Conclusions}

Based on the local shell geometry in the region of the axisymmetric dimple, experimental buckling loads were found to be in rather good agreement with numerical calculations using an exact model formulation including the effects of a clamped edge constraint and arbitrary imperfection amplitudes and wavelengths, The asymptotic formula of Amazigo and Budiansky specialized to the cosine dimple, gives quite reasonable predictions for values of the imperfection-thickness ratio even as large as 0.5 . In the critical wavelength range (i.e., $0.5 \leq$ $\left.l_{x} / l_{F} \leq 1.5\right)$ it is conservative. In general, for the axisymmetric dimple profile considered, buckling load reductions are not as severe as compared to a sinusoidal distribution extending over the entire cylinder length. However, it is quite apparent that very small axisymmetric dimple imperfections drastically reduce the buckling load of a circular cylindrical shell in axial compression.

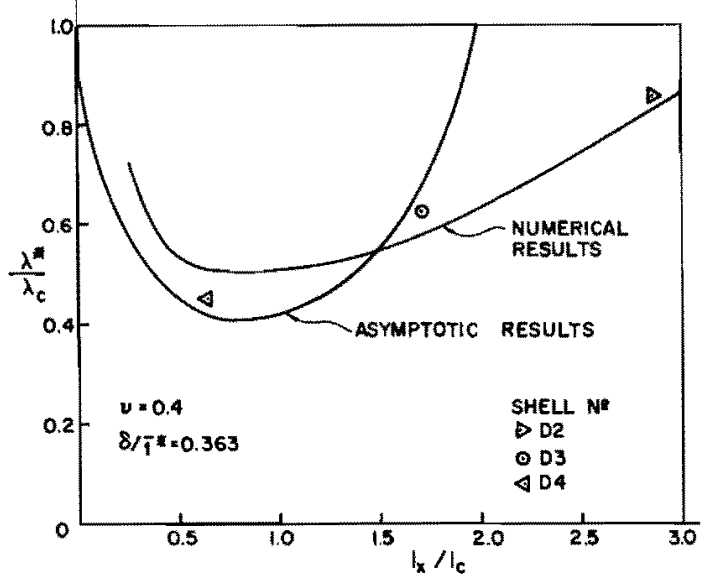

Fig. 8 Buckling load of a circular cylindrical shell vs axisymmetric dimple imperfection wavelength for a particular value of imperfection amplitude.
Fig. 7 Buckling load of a circular cylindrical shell vs axisymmetric dimple imperfection amplitude for a par-

ticular value of imperfection wavelength.

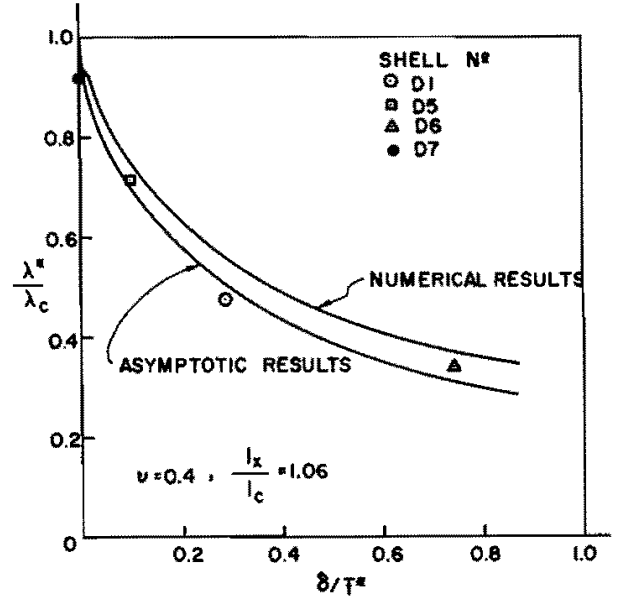




\section{References}

"Almroth, B. O., "Influence of Edge Conditions on the Stability of Axially Compressed Cylindrical Shells," CR-161, Feb. 1965, NASA; also AIAA Journal, Vol. 4, No. 1, Jan. 1966, pp. 134-140.

"Tennyson, R. C., "Photoelastic Circular Cylinders in Axial Compression," STP 419, 1967, American Society for Testing and Materials.

'Tennyson, R. C. and Muggeridge, D. B., "Buckling of Axisymmetric Imperfect Circular Cylindrical Shells under Axial Compression," AIAA Journal, Vol. 7, No. 11, Nov. 1969, pp. $2127-2131$.

'Babcock, C. D. and Sechler, E. E., "The Effect of Initial Imperfections on the Buckling Stress of Cylindrical Shells," TN D-2005, NASA, July 1963.

'Arbocz, J. and Babcock, C. D., "The Effect of General Imperfections on the Buckling of Cylindrical Shells," Transactions of ASME, Ser. 36, E; Journal of Applied Mechanics, 1969, pp. $28-38$.

"Tennyson, R. C., "The Effects of Unreinforced Circular Cutouts on the Buckling of Circular Cylindrical Shells under Axial Compression," Transactions of ASME; Journal of Engineering for Industry, Nov, 1968.
${ }^{7}$ Brogan, F. and Almroth, B. O., "Buckling of Cylinders with Cutouts," AIAA Journal, Vol. 8, No. 2, Feb. 1970, pp. 236-240.

"Babcock, C. D., "The Influence of a Local Imperfection on the Buckling Load of a Cylindrical Shell Under Axial Compression," GALCIT SM 68-4, Feb. 1968, California Institute of 'Technology, Pasadena, Calif.

- Amazigo, J. C. and Budiansky, B., private communication, 1969, Harvard Univ., Cambridge, Mass.

io Koiter, W. T., "On the Stability of Elastic Equilibrium," thesis, 1945; Delft, H. J. Paris, Amsterdam; also NASA Technical Translation F-10, 833, March 1967.

"Koiter, W. T., "The Effect of Axisymmetric Imperfections on the Buckling of Cylindrical Shells under Axial Compression," Kominklijke Nederlandse Akademie van Wetenschappen, Proceedings B66, 1963, pp. 265-279.

${ }_{12}$ Almroth, B. O., "Influences of Imperfections and Edge Restraint on the Buckling of Axially Compressed Cylinders," CR-432, April 1966, NASA.

1a Schiffner, K." "Untersuchung des Stabilitätsverhaltens dünnwandiger Kegelschalen unter axialsymmetrischer Belastung mittels einer nichtlinearen Schalentheorie," Jahrbuch 1965 der WGLR, pp. 448-453.

"Arbocz, J., "Buckling of Conical Shells under Axial Com"pression," CR-1162, 1968, NASA. 